Robert Ménard

\section{ADRESSES}

R. Ménard: Laboratoire de biologie et génétique du paludisme, Institut Pasteur, 28, rue du Docteur-Roux, 75724 Paris Cedex 15, France. e-mail : rmenard@pasteur.fr. A. Scherf: Unité de biologie des interactions hôte-parasite, Institut Pasteur, 28, rue du Docteur-Roux, 75724 Paris Cedex 15, France.

TIRÉS À PART

\title{
L'impact de la génétique inverse dans l'étude de la biologie de Plasmodium et de la physiopathologie du paludisme
}

L'étude de Plasmodium, le parasite responsable du paludisme, a été limitée dans le passé par la complexité de son cycle biologique et la rareté des outils, en particulier génétiques. Au cours de ces dernières années, des techniques de génétique inverse, qui permettent de manipuler les gènes et d'étudier la fonction de leur produit, ont été développées. Ces techniques arrivent au moment où la séquence du génome de l'espèce la plus pathogène pour l'homme est déjà presque entièrement connue, et où débute le séquençage du génome d'espèces plasmodiales modèles. Ces avancées technologiques promettent d'avoir un impact rapide sur notre compréhension de la physiopathologie du paludisme.

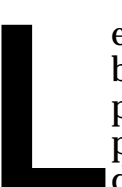

e paludisme reste un problème majeur de santé publique. Les derniers rapports de l'Organisation Monliale de la Santé (OMS) indiquent que près d'un milliard de personnes dans le monde sont porteuses du parasite causal, Plasmodium spp. Chaque année, le parasite cause environ 500 millions d'épisodes fébriles et entraîne la mort de près de trois millions d'individus, le plus souvent des enfants âgés de moins de cing ans. Plus du tiers de la population mondiale est à risque, et, dans la majorité des régions infestées, le parasite a développé des mécanismes de résistance à la plupart des médicaments disponibles [1].

Plasmodium est un protozoaire, eucaryote unicellulaire. Parmi les centaines d'espèces de Plasmodium décrites, quatre infectent I'homme dont une seule, Plasmodium falciparum, peut entraîner la mort. La vie de Plasmodium est un cycle qui se déroule chez deux hôtes, un hôte vertébré et un moustique du genre anophèle (figure 1). Chez I'hôte vertébré, seule l'infection des érythrocytes est symptomatique. Les symptômes, les classiques accès fébriles et l'anémie, sont dus avant tout aux cycles répétés de pénétration-multiplica- 


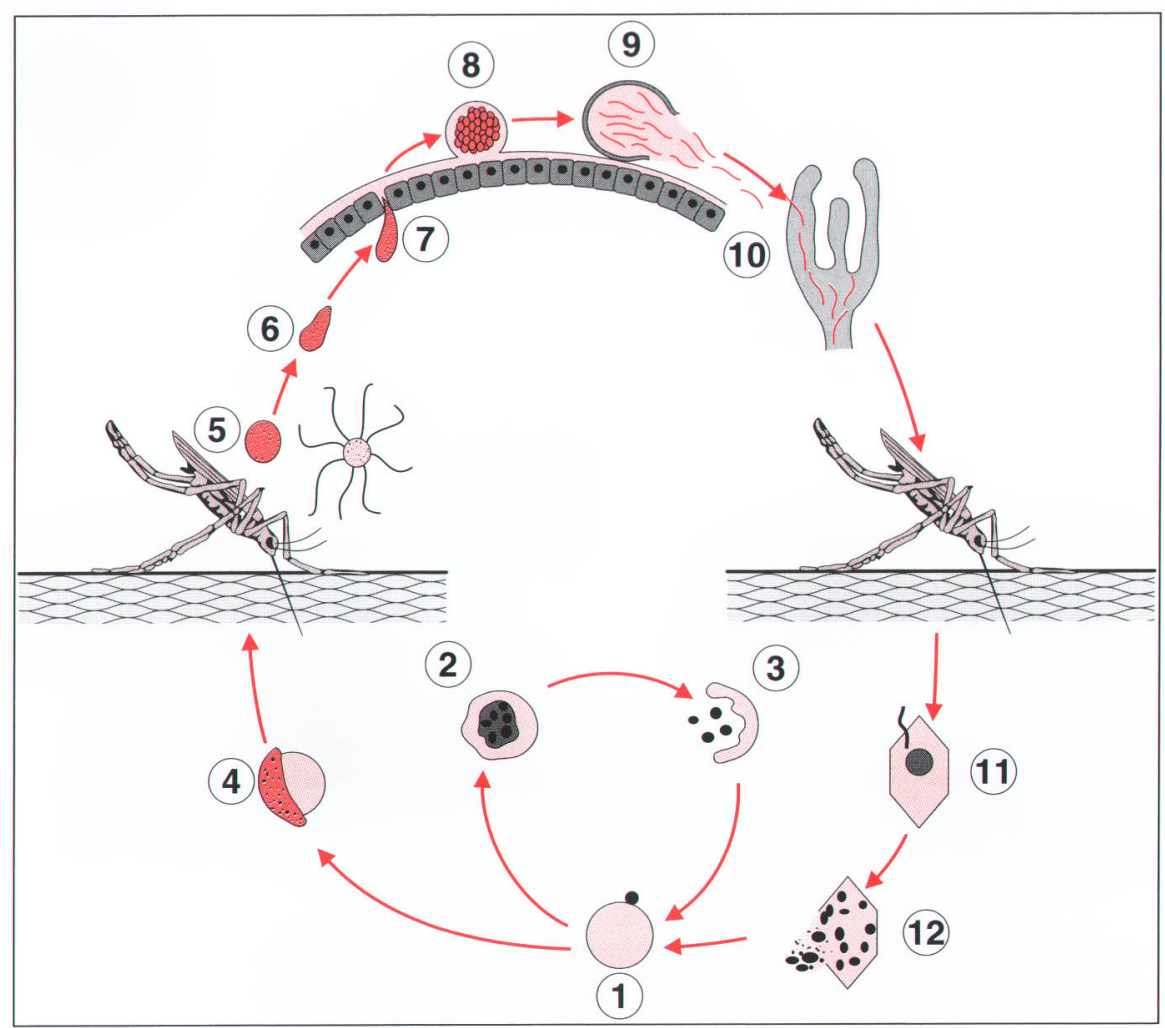

Figure 1. Cycle de vie de Plasmodium. Le cycle du parasite se déroule chez deux hôtes: un hôte définitif, un moustique du genre anophèle, et un hôte intermédiaire, un vertébré. Au cours de ce cycle, le parasite prend de nombreuses formes. Les mérozoïtes envahissent les érythrocytes (1) et se multiplient en 10 à 20 nouveaux mérozoïtes (2); chacun des mérozoïtes produits, après libération dans le courant circulatoire (3), peut alors infecter un nouvel érythrocyte (cycle de réplication asexué). À chaque cycle érythrocytaire, une proportion des parasites intra-érythrocytaires se différencient en formes sexuées, ou gamétocytes (4), qui ne poursuivront leur développement qu'une fois ingérées par un moustique au cours d'un repas sanguin. Dans l'intestin du moustique, les gamétocytes sont libérés, se différencient en gamètes (5) et fusionnent en un zygote diploïde (6). Celui-ci se transforme rapidement en un ookinète (7). L'ookinète traverse alors la matrice péritrophique et l'épithélium du mésentéron du moustique pour s'installer entre la couche de cellules épithéliales et la lame basale, et se transforme en un oocyste (8). La maturation d'un oocyste engendre, au bout de 5 à 10 jours, 5000 à 10000 sporozoïtes haploïdes. Ceux-ci sont libérés (9) dans I'hémocœle du moustique puis transportés jusqu'aux glandes salivaires par l'hémolymphe, le fluide qui baigne I'hémocœle. Les sporozoïtes traversent les cellules acinaires des glandes salivaires puis le canal salivaire (10), et peuvent alors être injectés à un nouvel hôte vertébré au cours du prochain repas sanguin. Lorsqu'un moustique infecté prend son repas sanguin sur un hôte vertébré, quelques dizaines de sporozoïtes sont injectés puis rapidement captés par le foie. Les sporozoïtes pénètrent dans les hépatocytes (11), et s'y multiplient (pendant 48 h à 1 semaine selon l'espèce plasmodiale) en des dizaines de milliers de mérozoïtes. Ceux-ci sont relâchés (12) par les hépatocytes, puis infectent les érythrocytes (1).

tion-libération des parasites (cycles de 24 à 72 heures selon les espèces), et à la lyse érythrocytaire qu'ils entraînent. La plupart des complications aiguës, en particulier le paludisme cérébral, sont dues à la modification de l'adhérence des
Le cycle de vie du parasite l'amène à interagir avec d'autres types cellulaires chez les deux hôtes (figure 1). A chaque cycle érythrocytaire, une partie des parasites cessent de se multiplier en se transformant en formes sexuées intra-érythrocytaires, qui seront ingérées par un moustique au cours d'un repas de sang. Les zygotes formés dans la lumière digestive du moustique doivent alors rapidement traverser l'épithélium intestinal pour produire de nouvelles formes parasitaires, les sporozoïtes, dans l'hémocœle (cavité) du moustique. Les sporozoïtes doivent ensuite traverser les glandes salivaires du moustique, et, après transmission à un nouvel hôte vertébré, envahir les hépatocytes. Dans ces cellules sont formées de nouvelles formes du parasite, les mérozoïtes, qui seront relâchés et infecteront les érythrocytes. A chaque phase du cycle, le parasite prend une forme caractéristique et exprime à sa surface des produits spécifiques, adaptés à la prochaine interaction avec I'hôte.

\section{Une nouvelle ère dans l'étude de la biologie du parasite}

Notre compréhension des interactions hôte-Plasmodium à un niveau moléculaire reste très limitée. Jusqu'au milieu des années 1990, seul un petit nombre de protéines parasitaires avaient été identifiées. Celles-ci étaient essentiellement des produits exprimés pendant la phase sanguine du cycle, la seule phase qui engendre de grandes quantités de parasites et se prête à une étude in vitro aisée [2]. Une dizaine de produits de stades sanguins du parasite ont été largement étudiés, aux plans épidémiologique, biochimique et surtout vaccinal. Malgré la quantité de données accumulées sur ces produits, leur fonction restait toutefois le plus souvent mal comprise, n'ayant pu être approchée que de façon indirecte ( $p a r$ immunomarquages et tests d'inhibition à l'aide d'anticorps ou de peptides).

érythrocytes infectés aux cellules endothéliales des veinules post-capillaires. L'interaction complexe entre le parasite et l'érythrocyte est donc naturellement au centre de la plupart des travaux de recherche sur le paludisme.
Lors des cing dernières années, des avancées technologiques majeures ont considérablement élargi les possibilités d'investigation sur la biologie du parasite. D'une part, I'amélioration des techniques de clonage de 
I'ADN de Plasmodium (dont la richesse moyenne en AT est de $80 \%$ et approche $100 \%$ sur de longs segments), et de son séquençage (par shotgun), ont déjà permis la publication de la séquence de 2 des 14 chromosomes de $P$. falciparum $[3,4]$. Le séquençage des 12 autres chromosomes est bien avancé, et près de $90 \%$ des séquences codantes de ce parasite (génome de 30 mégabases) sont déjà disponibles dans les bases de données. De nombreux projets de séquençage de fragments d'ADNC ont été entrepris chez diverses espèces et à divers stades de Plasmodium, et il est prévu que le génome d'au moins une ou deux autres espèces de Plasmodium soit séquencé en totalité. Par ailleurs, les premiers succès de transfection de Plasmodium ont été obtenus en 1995, chez P. falciparum [5] puis chez $P$. berghei [6], une espèce qui infecte les rongeurs de laboratoire et constitue le modèle d'étude de choix des stades sporozoïtes et intrahépatocytaires du parasite. Les premières démonstrations de recombinaison homologue ont rapidement suivi $[7,8]$, ouvrant la voie à la création de mutations dirigées chez les deux espèces $[9,10]$. II est donc maintenant possible d'envisager une étude fonctionnelle du génome plasmodial, et une étude moléculaire de la physiopathologie du paludisme.

\section{Plasmodium : un contexte génétique favorable pour la génétique inverse}

La démarche de génétique inverse part d'un gène d'intérêt pour aboutir a la fonction de son produit. Elle s'oppose à la démarche de génétique classique, qui part d'un phénotype d'intérêt pour remonter aux gènes qui le codent. La technique de choix pour caractériser la fonction in vivo d'une protéine est l'inactivation du gène correspondant par intégration dirigée d'ADN transformant dans le gène cible (figure $2 A, 2 B$ ). Chez Plasmodium, comme chez d'autres eucaryotes unicellulaires tels que la levure, l'intégration d'ADN transformant survient exclusivement par recombinaison homologue, ce qui facilite considérablement le clonage des mutants d'intérêt. Puisque Plasmodium est haploïde et que la plupart de ses

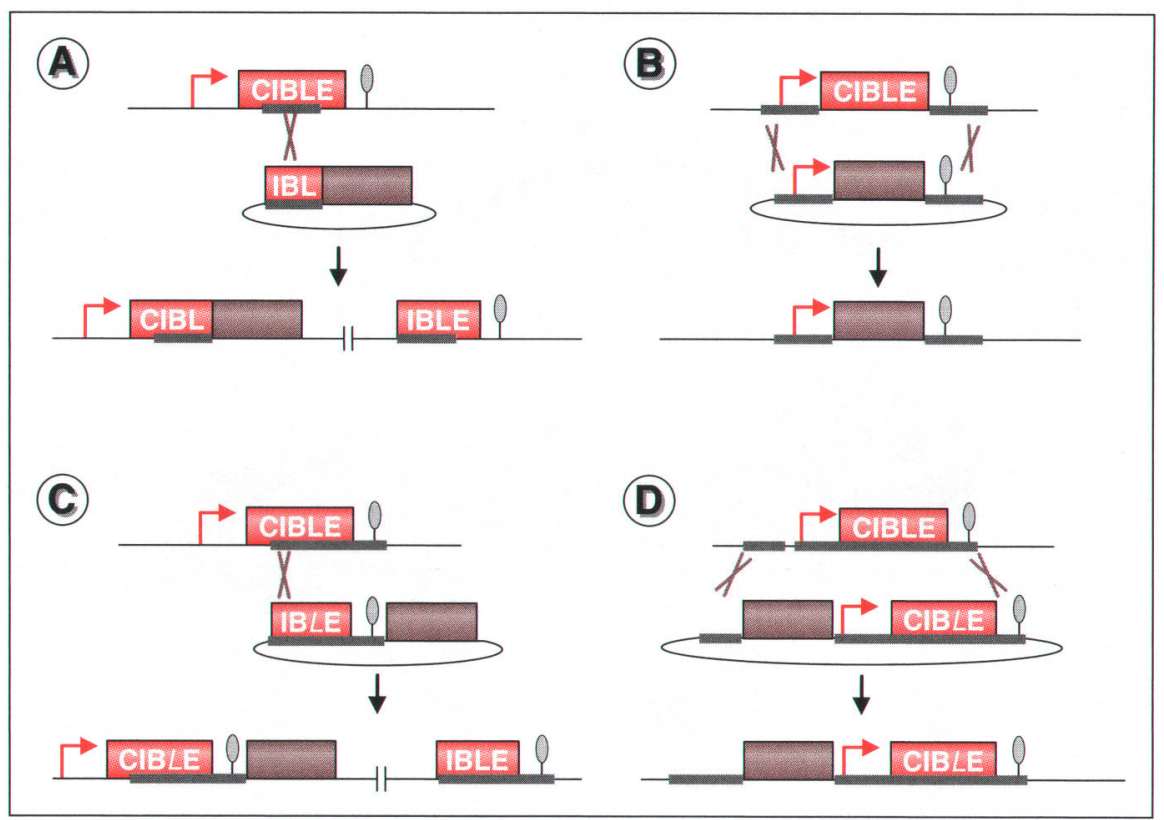

Figure 2. Inactivation ( $A$ et $B$ ) et modification ( $C$ et $D$ ) d'un gène par recombinaison homologue. La séquence codante du gène cible est représentée par une boîte, le promoteur par une flèche, les séquences 3' nécessaires à son expression par une ellipse, la cassette de résistance par une boîte sombre, les régions homologues par des lignes épaisses, et les événements de crossing over entre régions homologues par des croix. A. Inactivation d'un gène à l'aide d'un plasmide d'insertion. La linéarisation du plasmide dans la région d'homologie (fragment interne du gène) favorise l'intégration du plasmide dans le locus cible par simple crossing over. Le locus final comporte deux copies tronquées du gène cible. B. Inactivation d'un gène à l'aide d'un plasmide de remplacement. Après coupure aux extrémités distales des régions d'homologie dans le plasmide, un double crossing over remplace le gène sauvage par la copie mutagénisée (échange allélique). $\mathbf{C}$. Modification d'un gène à l'aide d'un plasmide d'insertion. Si le plasmide est linéarisé dans la région d'homologie en amont de la mutation (L), le locus final contient une copie du gène qui est de pleine longueur, modifiée et exprimée, et une seconde copie qui est tronquée et non exprimée. D. Modification d'un gène à l'aide d'un plasmide de remplacement. Après coupure aux extrémités distales des régions d'homologie dans le plasmide, un double crossing over permet l'échange de la copie sauvage du gène par la copie modifiée.

dans le génome, un seul événement de recombinaison homologue peut donc produire un clone dépourvu d'une protéine d'intérêt.

Sur le plan technique, seuls les stades sanguins de Plasmodium peuvent être transfectés (Tableau I). Cela implique que les recombinants ne peuvent être sélectionnés que si leur capacité d'envahir et de se multiplier dans les érythrocytes reste intacte. L'inactivation des gènes impliqués dans ces évé nements est donc impossible. En revanche, les gènes exprimés uniquement aux stades non érythrocytaires du parasite, même essentiels, peuvent être inactivés et leur phénotype analysé.

\section{Génétique inverse et stades non énythrocytaires du parasite}

\section{0 ocyste et formation des sporozoïtes}

La première protéine identifiée chez Plasmodium fut la protéine circumsporozoïte (CS) [11]. CS est la protéine majoritaire de surface des sporozoïtes, la forme parasitaire qui envahit les glandes salivaires du moustique, puis les hépatocytes du mammifère. Depuis les premières démonstrations que des anticorps dirigés contre la protéine CS peuvent 
Tableau I. Modèles d'étude génétique chez Plasmodium.

\begin{tabular}{|c|c|c|}
\hline Espèce & P. falciparum & P. berghei \\
\hline Hôte & Homme & Rongeurs \\
\hline \multicolumn{3}{|l|}{ Transfection } \\
\hline $\begin{array}{l}\text { Stades transfectés Stade } \\
\text { ADN transformant } \\
\text { Cycle intra-érythrocytaire } \\
\text { Sélection des recombinants } \\
\text { Temps de sélection d'un clone }\end{array}$ & $\begin{array}{l}\text { s intra-érythrocytaires } \\
\text { Circulaire } \\
48 \mathrm{~h} \\
\text { In vitro } \\
\sim 4-6 \text { mois }\end{array}$ & $\begin{array}{c}\text { Mérozoïtes } \\
\text { Circulaire, linéaire } \\
24 \mathrm{~h} \\
\text { In vivo } \\
\sim 4 \text { semaines }\end{array}$ \\
\hline \multicolumn{3}{|l|}{ Manipulations du génome } \\
\hline $\begin{array}{l}\text { Simple crossover } \\
\text { Double crossover }\end{array}$ & $\begin{array}{l}+ \\
-\end{array}$ & $\begin{array}{l}+ \\
+\end{array}$ \\
\hline \multicolumn{3}{|l|}{ Analyse phénotypique } \\
\hline $\begin{array}{l}\text { Cycle érythrocytaire in vitro } \\
\text { Cycle érythrocytaire in vivo } \\
\text { Adhérence El-CE } \\
\text { Stades infectant le moustique } \\
\text { Infection hépatocytaire in vitro } \\
\text { Infection hépatique in vivo }\end{array}$ & $\begin{array}{l}+ \\
- \\
+ \\
+- \\
+ \\
-\end{array}$ & $\begin{array}{l}- \\
+ \\
- \\
+ \\
+ \\
+\end{array}$ \\
\hline
\end{tabular}

1. L'ADN linéarisé n'engendre pas de recombinants; I'ADN circulaire ne permet l'isolement de recombinants (intégration dans le génome) qu'après de longs protocoles de sélection.

2. L'ADN circulaire permet d'obtenir des parasites portant des épisomes; I'ADN linéarisé permet la sélection rapide de recombinants (intégration dans le génome).

3. Adhérence entre les érythrocytes infectés et cellules endothéliales.

inhiber l'entrée des sporozoïtes dans les hépatocytes, chez le rongeur et chez l'homme [12, 13], la plupart des études sur la protéine ont concerné son pouvoir vaccinant. De nombreuses études fonctionnelles sur la protéine CS ont montré qu'elle est impliquée dans l'adhérence des sporozoïtes aux glandes salivaires du moustique via sa région amino-terminale [14], et aux héparane sulfate protéoglycanes (HSPG) des hépatocytes via sa région carboxy-terminale, un motif de type I de la thrombospondine (M1T) [15].

$L$ 'effet de l'inactivation du gène $C S$, obtenue chez $P$. berghei, fut une surprise: les parasites CS(-), au lieu d'engendrer comme attendu des sporozoîtes dépourvus de $C S$, ne produisent pas de sporozoïte [9]. Chez la souche sauvage, les sporozoïtes sont formés par bourgeonnement à la périphérie d'une forme large et multinucléée du parasite, l'oocyste, située dans la paroi de l'intestin du moustique (figure 1). CS est exprimée à la surface de l'oocyste et détermine les points de bourgeonnement des $\mathrm{m} / \mathrm{s} \mathrm{n}^{\circ} 6-7$, vol. 17 , juin-juillet 2001 apicomplexes (le plus important des protozoaires). Ils sont capables de pénétrer dans une cellule hôte en l'espace de quelques secondes par un mécanisme locomoteur (figure 3B) et de se déplacer en glissant sur tout substrat solide (figure $3 \mathrm{C}$ ). Pendant cette mobilité en glissant (gliding motility), la cellule apicomplexe ne change pas de forme et peut atteindre des vitesses considérables $(10 \mu \mathrm{m} / \mathrm{s})$. Une cellule animale ou un protozoaire amœboïde se déplacent au contraire en rampant (crawling motility), un type de mobilité qui met en jeu la formation continuelle de projections membranaires à l'avant de la cellule et des vitesses moindres $(0,001 \mu \mathrm{m} / \mathrm{s}$ pour un fibroblaste, et $1 \mu \mathrm{m} / \mathrm{s}$ pour la plus rapide des amibes). Mobilité en glissant et invasion cellulaire par les apicomplexes, cellules allongées et hautement polarisées, résultent d'un processus de capping antéro-postérieur de protéines de surface [16]. Des produits sont d'abord sécrétés sur la surface du pôle antérieur du parasite, à partir de vésicules cytoplasmiques spécialisées (voir légende de la figure $3 A$ ), et s'attachent au substrat ou à la surface cellulaire. Ils sont ensuite transloqués jusqu'au pôle postérieur du parasite, poussant ainsi le parasite en avant sur le substrat ou dans une vacuole intracellulaire (figure 3B).

Sur le plan moléculaire, on sait que ces deux processus dépendent de I'actine du parasite [17]. Le lien entre la polymérisation d'actine parasitaire et le mouvement rétrograde des interactions de surface reste obscur, mais implique probablement un moteur de type myosine. Un nouvelle pièce du puzzle est venue de l'analyse de TRAP (thrombospondin-related an on ymous protein [18]), une protéine transmembranaire de surface du sporozoïte plasmodial et sécrétée à son pôle antérieur. Son domaine extracellulaire contient deux modules d'adhérence aux cellules ou à la matrice extracellulaire: un module $M 1 T$, similaire à celui de la protéine $\mathrm{CS}$, et un domaine A du facteur von Willebrand (ou domaine I de nombreuses chaînes $\alpha$ d'intégrines). La plupart des études sur TRAP avaient conclu, comme pour CS, à son rôle dans le pouvoir d'adhérence des sporozoïtes aux cellules cibles [19]. Là encore, l'inactivation du gène a 


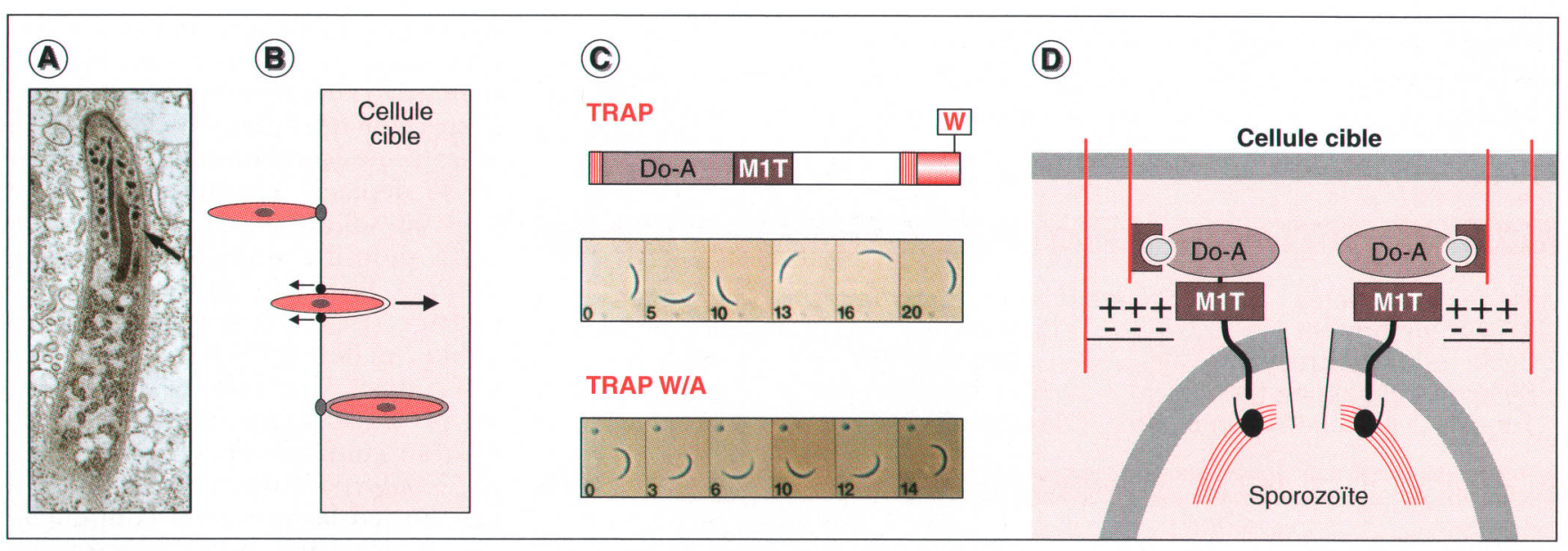

Figure 3. Mobilité en glissant et invasion cellulaire des sporozoïtes. A. Structure d'un sporozoïte de Plasmodium. Le sporozoïte possède les propriétés structurales typiques des stades invasifs d'apicomplexes: c'est une cellule allongée $(\sim 10 \mu \mathrm{m}$ de longueur) et polarisée, dont le pôle antérieur contient des vésicules sécrétoires appelées rhoptries (allongées) ou micronèmes (circulaires) qui sécrètent leur contenu à l'extrémité antérieure du parasite par un fin conduit. B. Invasion d'un sporozoïte dans une cellule hôte. Le sporozoïte forme une jonction entre son pôle antérieur et la surface de la cellule, qui est progressivement transloquée jusqu'à l'extrémité postérieure du parasite. Puisque la jonction est fixe, c'est le parasite qui est poussé dans la cellule à l'intérieur d'une vacuole parasitophore. La pénétration dépend d'une dynamique de l'actine du parasite, mais pas (ou peu) de la cellule hôte qui reste apparemment passive pendant l'entrée du parasite. C. Représentation schématique de la protéine TRAP et rôle dans la mobilité en glissant. TRAP possède un domaine extracellulaire qui comporte un domaine A de chaîne $\alpha$ d'intégrine (Do-A) et un motif de type 1 de la thrombospondine (MIT), et une partie cytoplasmique de $\sim 50$ résidus dont un tryptophane (W) carboxy-terminal. Sur une lame de verre, le sporozoïte glisse en décrivant un cercle toutes les 20 secondes (et en relâchant continuellement la portion extracellulaire de TRAP sur le substrat). La modification du résidu tryptophane carboxy-terminal de TRAP transforme le mouvement circulaire des sporozoïtes en un mouvement en pendule. Le mouvement en avant ( 0 -10 secondes) correspond à la longueur du sporozoïte ( $1 / 3$ de cercle), et à la translocation de TRAP de son pôle antérieur à son pôle postérieur; puis le sporozoïte s'arrête, probablement par manque de clivage de TRAP, et le sporozoïte revient alors à sa position initiale, avant que le cycle ne se répète. D. Le MIT et le Do-A de TRAP forment le système d'ancrage du sporozoïte à la cellule cible pendant l'entrée. Le MIT se lie aux chaînes glycosaminoglycanes des héparane sulfates de la surface cellulaire (via des interactions ioniques), et le Do-A se lie à un récepteur inconnu (liaison dépendant d'un ion divalent $\mathrm{Mg}^{2+}$ ou $\mathrm{Mn}^{2+}$ ). La force exercée par le système moteur cytoplasmique (représenté par l'actine -lignes parrallèles- et une myosine) peut être transmise via la partie cytoplasmique de TRAP à ces modules d'ancrage, permettant l'internalisation progressive du sporozoïte.

révélé une fonction différente du produit: TRAP n'intervient pas dans le pouvoir d'adhérence des sporozoïtes, mais est essentielle à leur pouvoir invasif dans les glandes salivaires du moustique et les hépatocytes du rongeur ainsi qu'à leur mobilité en glissant [20]. TRAP se présente donc comme un bon candidat pour lier le moteur cytoplasmique et les interactions extracellulaires au cours de la mobilité et de l'invasion cellulaire des sporozoïtes.

La mise au point de techniques de mutagen èse subtile chez $P$. berghei [21] (figures 2C, 2D) a permis de confirmer cette hypothèse. La délétion de la partie cytoplasmique de TRAP abolit toute mobilité/ invasion des sporozoïtes, et des modifications ponctuelles dans sa partie distale transforment le mouvement norma-

un mouvement pendulaire (figure $3 \mathrm{C}$ ) [22]. La mutagenèse du module M1T et du domaine A de TRAP a révélé que ces deux modules d'adhérence sont nécessaires au pouvoir invasif des sporozoïtes, mais pas à leur mobilité. Ils forment un double système d'ancrage à la surface de la cellule. Les pertes de fonction d'un seul domaine réduisent, mais n'abolissent pas, le pouvoir invasif des sporozoïtes (chez l'insecte comme chez la souris), alors que les pertes de fonction des deux domaines abolissent le pouvoir invasif toujours sans affecter la mobilité en glissant. Ce double système d'adhérence à la cellule apparaît donc comme le ligand majeur, et peut-être exclusif, de la jonction sur laquelle le parasite exerce une force pour se propulser dans la cellule (figure 3D). Ce double ligand assure l'entrée dans toute cel-
Iule cible, in vivo chez l'insecte et le rongeur, et in vitro quelle que soit la lignée.

Des protéines apparentées à TRAP ont été décrites chez de nombreux parasites apicomplexes, en particulier Toxoplasma et Cryptosporidium, responsables d'infections sévères chez le sujet immunodéprimé et le chez le nouveau-né. Elles portent une partie cytoplasmique de longueur semblable mais sans similarité de séquence, et des domaines extracellulaires portant divers arrangements de MIT et de domaines A. Deux arguments issus de la génétique inverse confirment que ces protéines sont des homologues fonctionnels de TRAP: (1) l'homologue chez le stade ookinète de Plasmodium, la protéine CTRP, est nécessaire à la mobilité des ookinètes et à leur tran sformation en oocystes (qui nécessite la traversée de 
I'épithélium intestinal du moustique) $[23,24]$; (2) I'homologue chez Toxoplasma, MIC2, est une protéine qui est co-localisée avec la jonction parasite-cellule transloquée pendant l'invasion [25] ; son domaine cytoplasmique est interchangeable avec celui de TRAP pour la mobilité et le pouvoir invasif des sporozoïtes de Plasmodium [22].

Ces études de génétique inverse suggèrent donc que le moteur cytoplasmique, un lien transmembranaire, et les ligands d'ancrage à la cellule sont conservés parmi les principaux apicomplexes pathogènes pour I'homme. Les études futures diront si les apicomplexes utilisent des systèmes moteurs acto-myosiniques distincts de ceux des cellules de l'hôte. Si tel est le cas, ils pourraient alors constituer une cible thérapeutique contre ce groupe important de parasites humains.

\section{Génétique inverse et stades érythrocytaires du parasite}

\section{Mérozoïte et invasion des érythrocytes}

$L$ 'entrée des mérozoïtes dans les érythrocytes, phénomène aisément observable in vitro, est le processus du cycle parasitaire qui a été le plus étudié sur les plans cellulaire et biochimique [26]. Le mérozoïte adhère d'abord à l'érythrocyte d'une façon aléatoire (random collision), puis s'oriente pour que son pôle apical se retrouve perpendiculaire à la surface de l'érythrocyte. La pénétration du mérozoïte dans une vacuole parasitophore survient alors par translocation typique d'une jonction serrée. De nombreuses molécules produites par les mérozoïtes ont été identifiées. Certaines sont présentes à leur surface, comme MSP-1 (merozoite surface protein I) [27]; d'autres sont localisées dans les vésicules sécrétoires du complexe apical (voir la légende de la figure 3A), comme EBA-175 (erythrocyte binding antigen 175) [28] et AMA1 (apical membrane protein 1) [30]. Dans la majorité des cas, des anticorps dirigés contre ces protéines altèrent le pouvoir invasif des mérozoïtes, faisant de beaucoup de ces protéines des candidats vaccins visant à interrompre la phase érythrocytaire $\mathrm{m} / \mathrm{s} \mathrm{n}^{\circ}$ 6-7, vol. 17 , juin-juillet 2001 du cycle. Au plan fonctionnel, la multiplicité des protéines parasitaires apparemment impliquées dans l'invasion des érythrocytes, ainsi que la diversité des voies d'invasion possibles [30], contrastent avec la situation décrite chez le sporozoïte.

Dans les stades érythrocytaires, les stades transfectables, I'inactivation d'un gène important est un événement létal. Une étude systématique, chez $P$. falciparum, de gènes considérés comme importants pour l'invasion des mérozoïtes a confirmé que la plupart ne pouvaient pas être inactivés [31]. Dans le cas de M SP-1, le produit est probablement essentiel puisque le gène ne peut être inactivé alors que des modifications du gène qui ne le détruisent pas sont possibles [32]. Pourtant, I'absence de sélection d'événement d'inactivation ne constitue pas une démonstration du caractère essentiel du produit, surtout chez $P$. falciparum, chez lequel les événements de recombinaison sont rares. Seules des techniques d'inactivation conditionnelle (promoteur inductible couplé à la production d'ARN anti-sens, ou recombinaison conditionnelle) pourront prouver le caractère essentiel d'un produit de mérozoïte, et surtout préciser sa fonction.

Dans certains cas, l'inactivation du gène n'a pas empêché la sélection des recombinants, et le produit n'est donc pas essentiel. Par exemple, des parasites dépourvus de MSP-3, une protéine de surface des mérozoïtes qui semble être la cible d'anticorps cytophiles protecteurs [33], ont pu être obtenus et leur phénotype ne diffère pas de celui de la souche sauvage [31]. De même, dans le cas de la protéine EBA-175, qui se lie à la glycophorine $A$ de la surface érythrocytaire de façon dépendante de l'acide sialique [34], des mutants ont été obtenus [35]. Ces mutants EBA175(-) empruntent une voie d'invasion indépendante de l'acide sialique, peut-être via les produits apparentés à EBA 175 dont l'existence a été révélée par le séquençage du génome de $P$. falciparum.

Il est probable que ces études par inactivation, ainsi que les études à venir de relations structure-fonction des protéines de mérozoïtes, auront des implications vaccinales importantes. Par exemple, dans le cas
d'EBA-175 qui n'est pas essentielle à la survie des parasites, son utilisation comme cible vaccinale pourrait favoriser la sélection de parasites (viables) dépourvus de la cible. Dans le cas de MSP-1, la protéine est probablement essentielle mais peut tolérer de grandes variations structurales (ou son échange par un homologue d'une autre espèce) sans altération de fonction [32], suggérant qu'un vaccin anti-MSP-1 puisse favoriser la sélection de mutants d'échappement. Une stratégie vaccinale visant à s'opposer à l'invasion des érythrocytes par les mérozoïtes devra comporter de nombreuses cibles vaccinales, et les arguments issus de la génétique inverse (conditionnelle ou non) seront certainement précieux dans le choix de ces cibles.

\section{Adhérence des érythrocytes infectés aux cellules endothéliales des microvaisseaux: un processus complexe}

Pendant la période de réplication intra-érythrocytaire, le parasite modifie la cellule hôte. Tout d'abord, la membrane de l'érythrocyte infecté devient plus perméable à des métabolites de faible poids moléculaire, probablement pour faciliter la croissance puis la multiplication du parasite [36]. Surtout, le parasite modifie les propriétés d'adhérence des érythrocytes infectés aux cellules endothéliales des veinules post-capillaires, ce qui aboutit à la séquestration des parasites dans les tissus profonds. $\mathrm{Ce}$ phénomène, qui a probablement évolué en réponse à la capacité de la pulpe rouge de la rate de l'hôte à détruire les érythrocytes anormaux/ infectés, est donc un facteur de survie des parasites. La séquestration entraîne des perturbations microcirculatoires et des dysfonctionnements métaboliques qui sont considérés comme directement responsables des complications aiguës du paludisme, en particulier le paludisme cérébral. Une perturbation structurale majeure de l'érythrocyte infecté est la formation de nombreuses petites protrusions denses aux électrons (knobs) à sa surface [37], qui permettent I'attachement de l'érythrocyte infecté à la cellule endothéliale (figure 4). Plusieurs produits parasitaires ont été localisés sous la mem- 


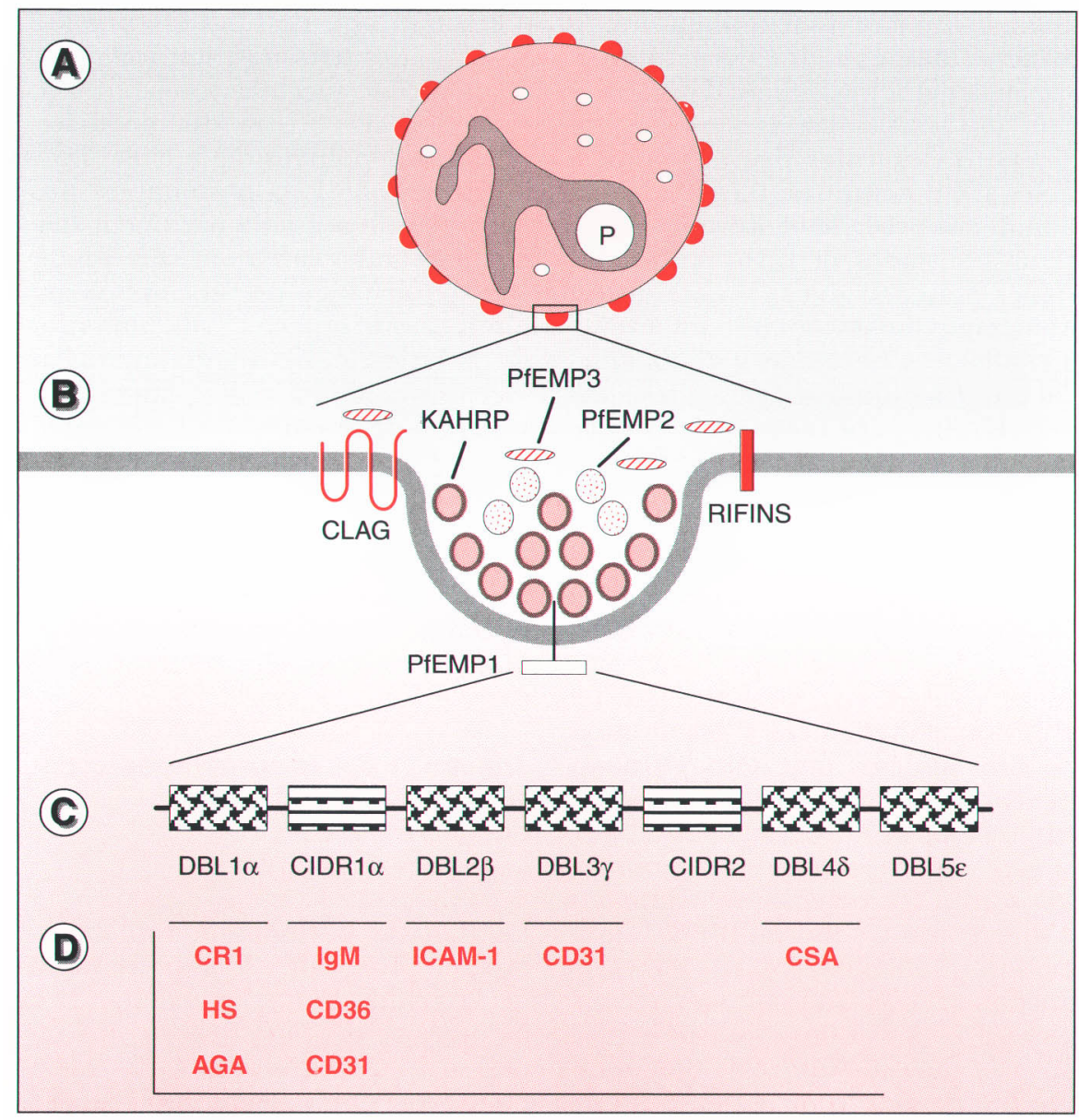

Figure 4. Un érythrocyte infecté par $\mathbf{P}$. falciparum et ses multiples voies d'adhérence à l'endothélium vasculaire. $\mathbf{A}$. Le parasite $(P)$, limité par la membrane de la vacuole parasitophore qui s'étend en un réseau membranaire tubulo-vésiculaire, entraîne la formation de protubérances à la surface de l'érythrocyte qui jouent un rôle majeur dans la séquestration des érythrocytes infectés dans la microcirculation profonde. B. Une protubérance contient plusieurs types de protéines parasitaires, notamment PfEMP1-3 et KAHRP. Le domaine carboxy-terminal, cytoplasmique, de PfEMP1 est conservé et ancre probalement la protéine à KAHRP ainsi qu'à divers composants du cytosquelette de l'érythrocyte comme l'actine et la spectrine. C. Le domaine amino-terminal, extracellulaire, de PfEMP1 comporte des modules d'adhérence dont la structure, le nombre, et l'arrangement diffèrent d'un variant à l'autre. Les deux modules de base sont nommés DBL (duffy binding-like domain, homologues aux domaines de la protéine EBA-175 des mérozoïtes) et CIRD (cysteine-rich interdomain region). D. Les récepteurs endothéliaux de ces modules incluent CR1: complement receptor 1; HS: héparane sulfates; AGA : antigène $A$ de groupe sanguin; CD31/PECAM-1: platelet endothelial cell adhesion molecule 1 ; ICAM-1: intercellular adhesion molecule 1 ; CSA : chondroïtine sulfate A. D'autres récepteurs comme VCAM1 (vascular cell adhesion molecule 1), ELAM-1 (endothelial leukocyte adhesion molecule 1), et la E-sélectine, ont aussi été impliqués. La combinaison spécifique des domaines exprimés à un moment donné de l'infection détermine le phénotype d'adhérence des érythrocytes infectés. D'autres protéines variantes sont exprimées à la surface de l'érythrocyte infecté, comme les produits CLAG (cytoadherence-linked asexual gene) et RIFINS, qui sont aussi codés par des familles multigéniques. Leur fonction reste inconnue.

brane plasmique de ces protrusions, comme la protéine KAHRP (knob- téine majoritaire, et les protéines PfEMP2 et PfEMP3 ( $P$. falciparum erythrocyte membrane proten 2 et 3$)$. Une quatrième protéine des protrusions, PfEMP1, possède un domaine cytoplasmique et un domaine extracellulaire composé d'une mosaïque de motifs d'adhérence à divers récepteurs de la cellule endothéliale (figure 4). Il existe dans le génome de $P$. falciparum une cinquantaine de gènes qui codent pour des protéines PfEMP1, appelés var, dont la structure générale est conservée mais dont l'agencement des motifs et les spécificités d'adhérence sont distincts (voir légende de la figure 4). Des anticorps spécifiques de variants peuvent protéger l'hôte, mais les parasites n'expriment qu'une protéine PfEMP1 à la fois et peuvent spontanément en changer à chaque cycle schizogonique de réplication $[38,39]$. Ce système de variation antigénique, dont le mécanisme est inconnu mais est certainement différent de celui bien décrit des trypanosomes [40], permet au parasite de maintenir une infection chronique en dépit de la pression immune de l'hôte.

Pourtant, le rôle des protrusions dans I'adhérence des érythrocytes infectés aux cellules endothéliales n'est pas clair, puisque toutes les souches parasitaires cultivées in vitro qui «cyto-adhèrent » $n$ 'engendrent pas de protrusions. L'inactivation du gène KAHRP [10] a montré: (1) que KH ARP est nécessaire à la formation des protrusions mais pas à l'expression en surface de la protéine PfEMP1; et (2) que les protrusions ne sont nécessaires à la cytoadhérence (au récepteur $C D 36$ ) que dans des conditions de flux qui miment celles des veinules post-capillaires. Une fonction de KAHRP et de la protrusion pourrait donc être de stabiliser la protéine PfEMP1, vraisemblablement en permettant l'ancrage de la protéine au cytosquelette de l'érythrocyte, pour une interaction solide. Les manipulations du gène PfEM P3 [41] ont montré que l'absence de la protéine n'affectait pas la formation de la protrusion, l'expression de PfEMP1 ou la cytoadhérence, mais que des portions tronquées de la protéine pouvaient abolir l'expression en surface de PfEMP1 et la cytoadhérence.

L'adhérence des érythrocytes infectés par $P$. falciparum aux récepteurs endothéliaux est un processus parti- 
culièrement complexe pour lequel le séquençage du génome du parasite ne cesse de proposer de nouveaux ligands. Les produits d'autres familles multigéniques que les gènes var, comme les gènes clag, stevor ou rifin, seraient aussi impliqués. D'une façon surprenante, I'inactivation d'un seul représentant de la famille clag, clag 9, affecte notablement la cytoadhérence [42]. De nombreuses autres études de génétique inverse, pouvant être associées à une technologie anti-sens, seront nécessaires pour clarifier le rôle de chacun de ces acteurs potentiels. Néanmoins, les premiers résultats permettent déjà d'entrevoir de possibles stratégies de protection. En effet, même si les adhésines sont la cible d'une variation antigénique, leur fonction dépend d'un complexe d'assemblage composé de produits plus conservés et codés par des gènes simple copie. Ces derniers pourraient donc être de meilleures cibles que les adhésines pour s'opposer à la cytoadhérence et aux accès de neuropaludisme, voire à d'autres manifestations de formes graves de paludisme.

\section{Perspectives}

La possibilité de manipuler le génome de Plasmodium marque une ère nouvelle dans l'étude de la biologie du parasite et de la physiopathologie du paludisme. La technique doit certes être simplifiée pour permettre sa diffusion à de nombreux laboratoires, et certains outils doivent encore être développés pour permettre l'analyse de tout produit plasmodial. Cependant, au vu du contexte génétique favorable (génome haploïde, gènes simple copie, recombinaison homologue exclusive) et des phénotypes défectueux évidents de la plupart des mutants obtenus jusqu'à présent, la génétique inverse promet d'être largement utilisée chez Plasmodium.

Maintenant qu'il est possible d'aller du gène à sa fonction, le prochain défi est de pouvoir remonter du phénotype aux gènes. Une telle démarche de génétique classique est à l'évidence nécessaire pour une étude complète des divers aspects de I'interaction hôte-parasite (pénétration puis développement dans les cellules hôtes, cytoadhérence des éry- throcytes parasités... ). Les approches par mutagenèse aléatoire et isolement de mutants défectueux, si utiles au décryptage des mécanismes de virulence des bactéries pathogènes, et récemment mises au point chez des protozoaires comme Leishmania et Toxoplasma $[43,44]$, n'ont pas été développées chez Plasmodium. Un système de mutagenèse au hasard serait de toute façon limité par des problèmes logistiques inhérents au cycle complexe du parasite (en particulier chez le moustique).

Il est clair que la séquence du génome, connue à plus de $90 \%$ pour P. falciparum, va constituer dans certains cas un crible puissant de gènes d'intérêt. Par exemple, la dissection progressive du métabolome du parasite a déjà permis de valider de nouvelles cibles thérapeutiques, en particulier des voies métaboliques d'un organite de type chloroplaste, l'apicoplaste $[45,46]$. Certains auteurs prédisent que la séquence du génome de $P$. falciparum permettra l'identification systématique d'épitopes B ou T et leur incorporation en "colliers de perles» dans un large ADN vaccinal, et une nouvelle stratégie de vaccination dite vaccinomique [47]. En revanche, il semble peu probable que la séquence suffise à identifier les produits impliqués dans les divers aspects de l'interaction avec les hôtes. En fait, presque tous les aspects de la vie de ces protozoaires parasites (invasion, séquestration, multiplication...) sont uniques, et il n'est sans doute pas surprenant que près de $60 \%$ des gènes de Plasmodium n'aient pas de similarité de séquence dans les bases de données ( soit deux fois plus que la plupart des organismes séquencés).

A défaut de cribles fondés sur la fonction des produits, restent ceux fondés sur l'expression des gènes. Les premières applications de la technologie des puces à ADN ont été rapportées chez Plasmodium, mais ne concernent pour l'instant que les stades érythrocytaires de $P$. falci parum $[48,49]$. Des techniques permettant l'analyse du transcriptome d'autres stades du parasite, produits en faibles quantités (en particulier les stades intrahépatocytaires), nécessiteront la mise au point de techniques d'amplification à partir de matériel trié par FACS ou capturé par microdissection par laser. Des techniques de clonage de messagers différentiellement exprimés par hybridation soustractive, et des techniques génétiques maintenant possibles de piégeage de promoteurs, seront certainement développées. La combinaison de ces approches devrait permettre une première sélection rationnelle, parmi les 7000 gènes du génome, de ceux qui pourraient intervenir dans un phénotype d'intérêt. Puisque la construction et la caractérisation d'un mutant restent une entreprise chez Plasmodium, les cribles devront être sélectifs. Le but est de pouvoir modéliser les étapes cruciales de processus pathogènes du parasite, en particulier pour $P$. falciparum, la seule espèce qui peut tuer I'homme. Les possibilités techniques sont malheureusement plus limitées chez cette espèce, les temps de sélection des mutants encore très longs et la biologie du parasite chez le moustique difficilement explorable. Certaines étapes de l'infection devront donc être étudiées avec d'autres espèces, comme P. knowlesi, qui infecte les primates simiens et peut être transfectée [50], ou P. berghei, qui infecte les rongeurs de laboratoire. Cette dernière espèce, dont le cycle complet peut être maintenu au laboratoire, est aussi un système génétique plus versatile et plus aisé. L'étude de l'interaction entre $P$. berghe et ses hôtes est sans limite, puisque non seulement le parasite mais aussi le moustique vecteur [51] et la souris sont génétiquement modifiables, et que la séquence du génome des trois partenaires va bientôt être disponible. Avec de tels outils, la biologie de Plasmodium va pouvoir être disséquée. Puisque cette biologie est unique, les études à venir devraient non seulement nous réserver des surprises, mais aussi déboucher sur des stratégies nouvelles de protection contre cet ennemi mortel

\section{Remerciements \\ Nous remercions Geneviève Milon, Teresa Gil Carvalho, Freddy Frieschknecht, et Hiroshi Sakamoto pour leurs remarques éclairées sur le manuscrit.}




\section{RÉFÉRENCES}

1. World Health Organisation. Fighting disease, fostering development. WHO report. Genève: OMS, 1996.

2. Trager W, Jensen JB. Human malaria parasites in continuous culture. Science 1976; 193: 673-5.

3. Gardner MJ, Tettelin H, Carucci DJ, et al. Chromosome 2 sequence of the human malaria parasite Plasmodium falciparum. Science 1998; 282 : 1126-32.

4. Bowman S, Lawson D, Basham D, et al. The complete nucleotide sequence of chromosome 3 of Plasmodium falciparum. Nature $1999 ; 400: 532-8$.

5. Wu Y, Sifri CD, Lei HH, et al. Transfection of Plasmodium falciparum within human red blood cells. Proc N atl A cad Sci USA 1995; 92: 973-7.

6. Van Dijk MR, Waters AP, Janse CI. Stable transfection of malaria parasite blood stages. Science 1995; 268: 1358-62.

7. Wu Y, Kirkman LA, Wellems TE. Transformation of Plasmodium falciparum malaria parasites by homologous integration of plasmids that confer resistance to pyrimethamine. Proc Natl Acad Sci USA 1996; 93: 1130-4.

8. Van Dijk MR, Janse CJ, Waters AP. Expression of a Plasmodium gene introduced into subtelomeric regions of Plasmodium berghei chromosomes. Science 1996; 271: 662-5.

9. Ménard R, Sultan AA, Cortes $C$, et al. Circumsporozoite protein is required for development of malaria sporozoites in mosquitoes. Nature 1997; 385: 336-40.

10. Crabb BS, Cooke BM, Reeder JC, et al. Targeted gene disruption shows that knobs enable malaria-infected red cells to cytoadhere under physiological shear stress. Cell 1997; 89: 287-96.

11. Dame JB, Williams JL, McCutchan TF, et al. Structure of the gene encoding the immunodominant surface antigen on the sporozoite of the human malaria parasite Plasmodium falciparum. Science 1984; 225 : 593-9.

12. Potocnjak $P$, Yoshida N, Nussenzweig RS, Nussenzweig $V$. Monovalent fragments (Fab) of monoclonal antibodies to a sporozoite surface antigen ( $\mathrm{Pb} 44)$ protect mice against malarial infections. J Exp M ed 1980; 151: 1504-13.

13. Sinnis $P$, N ussenzweig V. Preventing sporozoite invasion of hepatocytes. In: Hoffman SL, ed. M alaria vaccine development: a multi-immune response approach. Washington DC, ASM Press, 1996: 15-33.

14. Sidjanski SP, Vanderberg J, Sinnis P. An opheles stephen si salivary glands bear receptors for region I of the circumsporozoite protein of Plasmodium falciparum. M ol Biochem Parasitol 1997; 90 : 33-41.

15. Cerami $C$, Frevert $U$, Sinnis $P$, et al. The basolateral domain of the hepatocyte plasma membrane bears receptors for the circumsporozoite protein of Plasmodium fal-
16. Russell DG. Host cell invasion by Apicomplexa: an expression of the parasite's contractile system? Parasitology 1983; 87 : 199-209.

17. Dobrowolski JM, Sibley LD. Toxoplasma invasion of mammalian cells is powered by the actin cytoskeleton of the parasite. Cell 1996; 84 : 933-9.

18. Robson $\mathrm{KJ}, \mathrm{H}$ all JR, Jennings $M W$, et al. $A$ highly conserved amino-acid sequence in thrombospondin, properdin, and in proteins from sporozoites and blood stages of a human malaria parasite. Nature 1988; 335: 79-82.

19. Müller HM, Reckmann I, Hollingdale $M R$, \& al. Thrombospondin related anonymous protein (TRAP) of Plasmodium falciparum binds specifically to sulfated glycoconjugates and to HepG 2 hepatoma cells suggesting a role for this molecule in sporozoite invasion of hepatocytes. EM BO J 1993; 12: 2881-9.

20. Sultan $A A$, Thathy $V$, Frevert $U$, et al. TRAP is necessary for gliding motility and infectivity of Plasmodium sporozoites. Cell 1997; 90: 511-22

21. Nunes A, Thathy $V$, Bruderer $T$, et al. Subtle mutagenesis by ends-in recombination in malaria parasites. M ol Cell Biol 1999; 19: 2895-902.

22. Kappe S, Bruderer T, Gantt S, et al. Conservation of a gliding motility and cell invasion machinery in apicomplexa parasites. J Cell Biol 1999; 147: 937-43.

23. Yuda M, Sakaida H, Chinzei Y. Targeted disruption of the Plasmodium berghei CTRP gene reveals its essential role in malaria infection of the vector mosquito. J Exp $M$ ed 1999; 190: 1711-5.

24. Dessens JT, Beetsma AL, Dimopoulos G, et al. CTRP is essential for mosquito infection by malaria ookinetes. EM B O 1999; 18: 6221-7.

25. Carruthers VB, Giddings OK, Sibley LD. Secretion of micronemal proteins is associated with Toxoplasma invasion of host cells. Cell M icrobiol 1999; 1 : 225-35.

26. Holder AA, Blackman MJ, Borre M, et al. Malaria parasites and erythrocyte invasion. Biochem Soc Trans 1994; 22 : 291-5.

27. Blackman MJ, Heidrich HG, Donachie $\mathrm{S}$, et al. A single fragment of a malaria merozoite surface protein remains on the parasite during red cell invasion and is the target of invasion-inhibiting antibodies. J Exp M ed 1990; 172: 379-82.

28. Kim BK. EBA-175: an erythrocyte-binding ligand of Plasmodium falciparum. Parasitol Today $1995 ; 11: 213-7$.

29. Peterson MG, Marshall VM, Smythe JA, et al. Integral membrane protein located in the apical complex of Plasmodium falciparum. Mol Cell Biol 1989; 9: 3151-4.

30. Dolan SA, Miller LH, Wellems TE. Evidence for a switching mechanism in the invasion of erythrocytes by Plasmodium falciparum. J Clin Invest 1990; 86: 618-24.

31. Cowman AF, Baldi DL, Healer J, et al. Functional analysis of proteins involved in
Plasmodium falciparum merozoite invasion of red blood cells. FEBS Lett 2000; 476: 84-8.

32. O'Donnell RA, Saul A, Cowman AF, $\mathrm{Crabb}$ BS. Functional conservation of the malaria vaccine antigen MSP-1 $1_{19}$ across distantly related Plasmodium species. Nat M ed $2000 ; 6 ; 91-5$.

33. O euvray C, Bouharoun-Tayoun H, Gras$M$ asse $H$, et al. Merozoite surface protein-3: a malaria protein inducing antibodies that promote Plasmodium falciparum killing by cooperation with blood monocytes. Blood 1994 ; 84 : 1594-602.

34. Camus D, Hadley TJ. A Plasmodium falciparum antigen that binds to host erythrocytes and merozoites. Science 1985; 230 : 553-6.

35. Reed MB, Caruana SR, Batchelor AH, et al. Targeted disruption of an erythrocyte binding antigen in Plasmodium falciparum is associated with a switch toward a sialic acidindependent pathway of invasion. Proc $\mathrm{N}$ atl Acad Sci USA 2000; 97 : 7509-14.

36. Deitsch KW, Wellems TE. Membrane modifications in erythrocytes parasitized by Plasmodium falciparum. M ol Biochem Parasitol 1996 ; $76: 1-10$.

37. Kilejian A. Characterization of a protein correlated with the production of knob-like protrusions on membranes of erythrocytes infected with Plasmodium falciparum. Proc Natl Acad Sci USA 1979; 76: 4650-3.

38. Scherf $A$, Hernandez-Rivas $R$, Buffet $P$, et al. Antigenic variation in malaria: in situ switching, relaxed and mutually exclusive transcription of var genes during intra-erythrocytic development in Plasmodium falciparum. EM BO J 1998; 17 : 5418-26.

39. Chen Q, Fernandez V, Sundstrom A, et al. Developmental selection of var gene expression in Plasmodium falciparum. Nature 1998 ; 394 : 392-5.

40. Borst $P$, Rudenko $G$. Antigenic variation in African trypanosomes. Science 1994; 264 : 1872-3.

41. Waterkeyn IG, Wickham ME, Davern $\mathrm{KM}$, \& al. Targeted mutagenesis of Plasmodium falciparum erythrocyte membrane protein 3 (PFEMP3) disrupts cytoadherence of malaria-infected red blood cells. EM BO J 2000; 19: 2813-23.

42. Trenholme KR, Gardiner DL, Holt DC, et al. Clag9: a cytoadherence gene in Plasmodium falciparum essential for binding of parasitized erythrocytes to CD 36. Proc Natl Acad Sci USA 2000; 97 : 4029-33.

43. Gueiros-Filho FJ, Beverley SM. Transkingdom transposition of the Drosophila element mariner within the protozoan Leishmania. Science 1997; 276: 1716-9.

44. Donald RG, Roos DS. Insertional mutagenesis and marker rescue in a protozoan parasite: cloning of the uracil phosphoribosyltran sferase locus from T oxoplasma gondii. Proc Natl A cad Sci USA 1995; 92: 5749-53.

45. Waller RF, Keeling PJ, Donald RG, et al. Nuclear-encoded proteinstarget to the plastid in Toxoplasma gondii and Plasmodium falciparum. Proc N atl Acad Sci USA 1998; 95: 12352-7. 


\section{RÉFÉRENCES}

46. Jomaa H, Wiesner J, Sanderbrand $\mathrm{S}$, et al. Inhibitors of the nonmevalonate path way of isoprenoid biosynthesis as antimalarial drugs. Science 1999 ; 285 : 1573-6.

47. Hoffman SL, Rogers WO, Carucci DJ, Venter JC. From genomics to vaccines: malaria as a model system. Nat M ed 1998; 4 : 1351-3.

48. H ayward RE, DeRisi JL, Alfadhli S, et al. Shotgun DNA microarrays and stage-specific gene expression in Plasmodiu m falciparum malaria. M ol M icrobiol 2000; 35: 6-14.

49. Ben Mamoun CB, Gluzman IY, Hott $C$, et al. Co-ordinated programme of gene expression during asexual intraerythrocytic development of the human malaria parasite Plasmodium falciparum revealed by microarray analysis. M ol M icrobiol 2001; 39: 26-36.

50. van der Wel AM, Tomas AM, Cocken C, et al. Transfection of the primate malaria parasite Plasmodium knowlesi using entirely heterologous constructs. J Exp Med 1997; 185: 1499-503.

51. Catteruccia F, Nolan T, Loukeris TG, et al. Stable germline transformation of the malaria mosquito Anopheles stephensi. Nature 2000; 405 : 959-62.

\section{Summary}

Reverse genetics, biology of Plasmodium and pathophysiology of malaria

Malaria remains one of the most devastating infectious diseases worldwide. In the past few years, reverse genetic techniques have been developed in Plasmodium, the etiological agent of malaria. Reverse genetics, which permits site-directed mutagenesis, is particularly straightforward in Plasmodium because its genome is haploid, most of its genes are singlecopy, and integration of exogenous constructs occurs exclusively via homologous recombination. Consequently, despite the complexity of the parasite life cycle, important insights into Plasmodium biology and host-parasite interactions have already been gained. In addition, the genome of various Plasmodium species is being sequenced, including that of $P$. falciparum, the species most deadly to humans. The combination of these technological breakthroughs should have a rapid and important impact on our understanding of the biology of Plasmodium and the physiopathology of malaria, and may ultimately lead to new ways to combat the parasite. 